\title{
Robotic radical prostatectomy in post HIFU salvage setting: tertiary center experience and review of the current literature
}

\author{
Stefano De Luca,"\#, Sabrina De Cillis, ${ }^{1, \#}$, Federico Piramide ${ }^{1}$, Paolo Alessio ${ }^{1}$, Filippo Russo ${ }^{2}$, Daniele \\ Amparore ${ }^{1}$, Gabriele Volpi ${ }^{1}$, Stefano Granato ${ }^{1}$, Matteo Manfredi ${ }^{1}$, Cristian Fiori ${ }^{1}$, Enrico Checcucci $^{13, \S}$, \\ Francesco Porpiglia ${ }^{1, \S}$ \\ 'Department of Oncology, Division of Urology, University of Turin, San Luigi Gonzaga Hospital, Orbassano, Turin 10043, Italy. \\ ${ }^{2}$ Department of Radiology, Candiolo Cancer Institute, FPO-IRCCS, Candiolo, Turin 10060, Italy. \\ ${ }^{3}$ Department of Surgery, Candiolo Cancer Institute, FPO-IRCCS, Candiolo, Turin 10060, Italy. \\ "These Authors equally contributed to the first authorship. \\ ${ }^{s}$ These Authors equally contributed to the senior authorship. \\ Correspondence to: Dr. Stefano De Luca, Department of Oncology, Division of Urology, University of Turin, San Luigi Gonzaga \\ Hospital, Orbassano, Turin 10043, Italy. E-mail: delucastefano@yahoo.it
}

How to cite this article: De Luca S, De Cillis S, Piramide F, Alessio P, Russo F, Amparore D, Volpi G, Granato S, Manfredi M, Fiori C, Checcucci E, Porpiglia F. Robotic radical prostatectomy in post HIFU salvage setting: tertiary center experience and review of the current literature. Mini-invasive Surg 2022;6:13. https://dx.doi.org/10.20517/2574-1225.2021.127

Received: 4 Nov 2021 First Decision: 10 Jan 2022 Revised: 25 Jan 2022 Accepted: 7 Feb 2022 Published: 24 Feb 2022

Academic Editor: Giulio Belli Copy Editor: Xi-Jun Chen Production Editor: Xi-Jun Chen

\begin{abstract}
Aim: The purpose of the study was to describe our surgical technique of salvage robot-assisted radical prostatectomy (SRARP) in patients who underwent primary high-intensity focused ultrasound (HIFU) and to report the perioperative, functional, and oncological outcomes during the first year follow up. The secondary aim of the study was to review the current literature evidence on this topic.
\end{abstract}

Methods: We retrospectively extracted, from our prospective RARP database, all the patients who underwent sRARP for biochemical recurrence after primary HIFU. All the surgical interventions were performed by a single surgeon following our total anatomical reconstruction (TAR) technique. Demographics, perioperative, functional, and oncological results were collected up to one year follow-up.

Results: Eleven patients underwent post-HIFU sRARP with TAR technique at our institution. All the surgical procedures were uneventful. All the complication recorded were classified as Clavien-Dindo Grade I. Continence rate at 1-, 3-, 6-, and 12-month post intervention was 36.3\%, 45.5\%, $63.6 \%$, and $81.1 \%$, respectively. Medium PSA 
at 12 months follow-up was $0.2 \mathrm{ng} / \mathrm{mL}$ (SD 0.01), with no Biochemical Failure (BCF) recorded.

Conclusion: SRARP with TAR technique is a safe and feasible procedure in patients with BCF after primary HIFU. No major complications were recorded, with good oncological and functional results after one year follow up.

Keywords: Prostate cancer, focal therapy, HIFU, safety, salvage therapy, magnetic resonance imaging, PSMA$\mathrm{PET} / \mathrm{CT}$

\section{INTRODUCTION}

The prostate cancer ( $\mathrm{PCa}$ ) screening strategies, mainly based on serum prostate-specific antigen (PSA) levels evaluation, currently result in an overdiagnosis of low-/intermediate-grade organ-confined neoplasms ${ }^{[1,2]}$. Some of these patients (i.e., low risk PCa with life expectancy $>10$ years) can be safely treated with active surveillance ${ }^{[3]}$. On the other hand, a number of patients with intermediate risk PCa are eligible for active radical treatment [i.e., radical prostatectomy (RP) or radiotherapy] which may be classified as "overtreatment" (mostly in case of low percentage of Gleason pattern $4, \leq 10 \%$ ), considering the potential side effects ${ }^{[4-6]}$.

This is the reason why urologists have started to offer a more tailored treatment in men with localized low and early intermediate grade PCa. The aim is to minimize the impact on patient's quality of life from primary surgery or radiotherapy while maintaining the best oncological outcomes ${ }^{[7]}$. Non-surgical focal therapies for PCa perfectly fit in this scenario since they are characterized by limited systemic effects and low chance of damaging the surrounding anatomical structures (i.e., urethra, sphincter, neurovascular bundles, and bladder neck $)^{[8-10]}$. On the other hand, considering that PCa is often multi-focal, a proportion of patients will develop local recurrent disease.

High-intensity focused ultrasound (HIFU) is one of the ablative modalities that have been approved for clinical use within prospective registry studies, although it remains experimental according to international guidelines $^{[3]}$. The percentage of local recurrence after HIFU is quite common mostly in case of partial compared to total ablation; $32 \%-40 \%$ experienced recurrence requiring further treatment within 5 years after partial HIFU ablation and $54 \%$ by 8 years in two large, multicentre series ${ }^{[9,11]}$. Furthermore, the rate of recurrence post-HIFU may increase with longer follow-up.

Despite no consensus on the optimal management of recurrent PCa after primary HIFU therapy, up to now salvage prostatectomy (sRP) is reserved for only very few patients because of technical challenges and frequent post-operative complications. In particular, there are very limited published data on functional and oncological outcomes for salvage robot assisted RP (sRARP) after HIFU ${ }^{[12-15]}$.

The primary aim of the present study was therefore to describe our surgical technique of sRARP characterizing the perioperative, functional, and oncological outcomes during the first year follow up. The secondary aim was to present a review of the current literature about this topic.

\section{METHODS}

\section{Study population}

We retrospectively evaluated our prospectively maintained RARP database and extracted patients who underwent sRARP with total anatomical reconstruction (TAR) technique for biochemical recurrence after primary HIFU from January 2015 to June 2020. Inclusion criteria were preoperative diagnosis of recurrent 
PCa with positive PSMA positron emission tomography scan and/or suspect area at prostate multiparametric magnetic resonance imaging (mpMRI). The radiologic suspect criteria for prostate lesions were defined a posteriori, in accordance with the Prostate Imaging Recurrence Reporting (PI-RR) score ${ }^{[16]}$. All the patients enrolled in the study had no contraindications in performing RARP (i.e., good performance status, no previous major abdominal surgery, and no contraindication in maintenance of Trendelemburg position).

Biochemical recurrence was defined, in accordance with ASTRO-Phoenix criteria, as a PSA rise equal or greater than $2 \mathrm{ng} / \mathrm{mL}$ above the PSA nadir, regardless of ongoing androgen deprivation therapy ${ }^{[17]}$. Patients with a follow up after salvage RARP less than 1 year were excluded.

\section{Surgical technique}

All the surgical interventions were performed by a single expert laparoscopic surgeon (F.P.) with great experience in robot-assisted PCa surgery. All the procedures were performed following the TAR technique which has been previously described and proven to be safe and feasible in different scenarios ${ }^{[18-20]}$. Briefly, after the demolition phase of the radical prostatectomy, a three-layer posterior reconstruction and a twolayer anterior reconstruction were performed in addition to the urethra-vesical anastomosis. As shown in Figure 1, the posterior reconstruction involved the Denonvilliers' fascia and the median raphe, then the retrotrigonal fascia and the median raphe, and lastly the bladder neck and the posterior aspect of the rhabdosphincter. The anterior reconstruction involved the muscular fibres of the bladder and the periurethral tissue, after a second layer of suture is performed between the vesical apron and the portion of the endopelvic fascia that covers the dorsal vein complex while involving the pubo-prostatic ligaments. An extended lymph node dissection was performed in all the cases including iliac, obturator, and presacral lymph node removal. Nerve-sparing was performed in very few cases, in accordance with preoperative local imaging staging and preoperative patient's erectile function.

\section{Data collection and statistical analysis}

Demographics were collected. In particular, pre-HIFU Gleason score, and D'Amico risk classification were evaluated, along with pre sRARP PSA levels and PI-RR score of mpMRI findings. PSA levels at 1, 3, 6, and 12 months after the intervention were registered.

Clavien Dindo classification system was used to classify postoperative complications up to 12 months. Baseline International Prostatic Symptoms Score, quality of life, and International Index of Erectile Function (IIEF-5) were collected and re-evaluated at 1, 3, 6 and 12 months after the procedure. Continuous variables were described using mean and standard deviation, and discrete variables were described using median and interquartile ranges. Frequency tables were used to summarize categorical variables.

\section{RESULTS}

From January 2015 to June 2020, 11 patients underwent post-HIFU sRARP with TAR technique at our institution. Demographics as well as preoperative and postoperative oncological and functional data are reported in Table 1 .

Of these 11 patients, 8 had previously undergone total gland ablation while 3 had partial gland ablation for low-intermediate risk PCa. The mean time interval between HIFU and sRARP was 32.3 months (SD 11.4 months). Mean pre sRARP PSA was $3.6 \mathrm{ng} / \mathrm{mL}$ (SD $2 \mathrm{ng} / \mathrm{mL}$ ) and mean prostate volume was $37.9 \mathrm{~mL}$ (SD $13.3 \mathrm{~mL})$. 
Table 1. Perioperative, pathological and follow up variables of the SRARP group

\begin{tabular}{|c|c|}
\hline & Total \\
\hline$n$ patients & 11 \\
\hline Age, mean (SD), years & $72.6(3.2)$ \\
\hline BMI, mean (SD) & $25.1(1.6)$ \\
\hline Pre-HIFU PSA, ng/dL; mean (SD) & $9.1(2.3)$ \\
\hline $\begin{array}{l}\text { Pre-HIFU Gleason score, number (\%) } \\
\cdot 6 \\
\cdot 7(3+4) \\
\cdot 7(4+3)\end{array}$ & $\begin{array}{l}5(45.5) \\
4(36.3) \\
2(18.1)\end{array}$ \\
\hline $\begin{array}{l}\text { Pre-RT risk; } \mathrm{N}^{\circ}(\%) \\
\text { - Low } \\
\text { - Intermediate }\end{array}$ & $\begin{array}{l}5(45.5) \\
6(55.5)\end{array}$ \\
\hline Pre-sRARP PSA, ng/dL; mean (SD) & $3.6(2.0)$ \\
\hline Pre-sRARP prostate volume, CC; mean (SD) & $37.9(13.3)$ \\
\hline Pre-sRARP IPSS; median (IQR) & $10(6.32-13.3)$ \\
\hline Pre-sRARP QoL; media (IQR) & $3(0-4)$ \\
\hline Pre-sRARP IIEF; median (IQR) & $16(11-23)$ \\
\hline $\begin{array}{l}\text { Pre-sRARP mpMRI, PiRads; } n \text { (\%) } \\
\cdot 3 \\
\cdot 4 \\
\cdot 5\end{array}$ & $\begin{array}{l}2(18.1) \\
6(54.5) \\
3(27.2)\end{array}$ \\
\hline Operative time, min; mean (SD) & $110(25.8)$ \\
\hline Catheterization time, days; median (IQR) & $5(4-7)$ \\
\hline Hospital stay, days; median (IQR) & $6(6-8)$ \\
\hline $\begin{array}{l}\text { Postoperative complications according to Clavi } \\
\text { - Grade 1: } \\
\text { o Fever } \\
\text { - Asymptomatic lymphocele }\end{array}$ & $\begin{array}{l}1(0) \\
1(0)\end{array}$ \\
\hline $\begin{array}{l}\text { Post-sRARP Gleason score, number }(\%) \\
\cdot 6 \\
\cdot 7(3+4) \\
\cdot 7(4+3)\end{array}$ & $\begin{array}{l}2(18.1) \\
6(55.5) \\
3(27.2)\end{array}$ \\
\hline Positive SM, number (\%) & $3(27.2)$ \\
\hline $\begin{array}{l}\text { Pathological stage, number (\%) } \\
\text { - pT2 } \\
\text { - pT3 }\end{array}$ & $\begin{array}{l}8(72.7) \\
3(2.2)\end{array}$ \\
\hline Numbers of lymph node removed; mean (SD) & $23.3(6.3)$ \\
\hline Pathological N1, number (\%) & $0(0)$ \\
\hline $\begin{array}{l}\text { Post-sRARP PSA, ng/dL; mean (SD) } \\
\cdot 1 \text { month } \\
\cdot 3 \text { months } \\
\cdot 6 \text { months } \\
\cdot 12 \text { months }\end{array}$ & $\begin{array}{l}0.02(0.01) \\
0.01(0.01) \\
0.03(0.02) \\
0.2(0.01)\end{array}$ \\
\hline $\begin{array}{l}\text { Post-sRARP continence rate; number (\%) } \\
\cdot 1 \text { month } \\
.3 \text { months } \\
\cdot 6 \text { months } \\
\cdot 12 \text { months }\end{array}$ & $\begin{array}{l}4(36.3) \\
5(45.5) \\
7(63.6) \\
9(81.1)\end{array}$ \\
\hline $\begin{array}{l}\text { Post-sRARP potency rate; number (\%) } \\
.1 \text { month } \\
.3 \text { months } \\
\cdot 6 \text { months } \\
\cdot 12 \text { months }\end{array}$ & $\begin{array}{l}0(0) \\
0(0) \\
1(9.0) \\
2(18.1)\end{array}$ \\
\hline
\end{tabular}

BMI: Body mass index; SD: standard deviation; PSA: prostate specific antigen; IQR: interquartile range; IPSS: international prostate symptoms Score; IIEF: international index of erectile function; QoL: quality of life; mpMRI: multiparametric magnetic resonance imaging; PiRADS: prostate imaging reporting and data system; sRARP: salvage robot assisted radical prostatectomy; SM: surgical margins. 


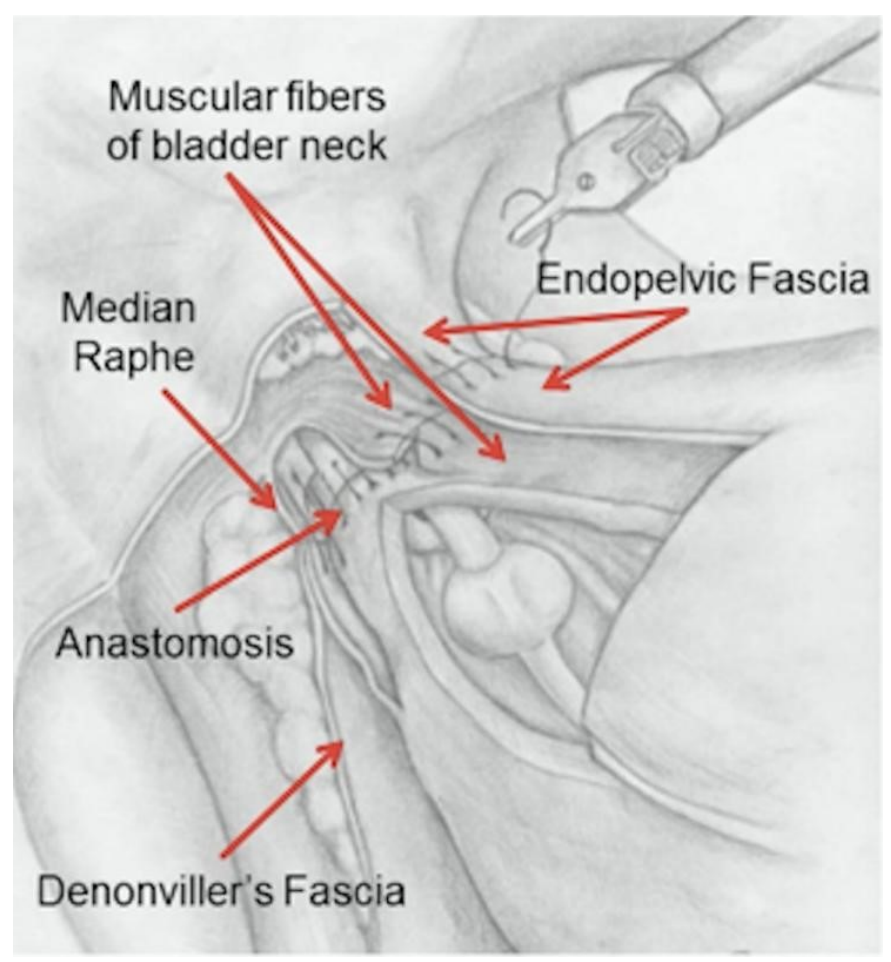

Figure 1. Total anatomical reconstruction technique: the posterior reconstruction is performed in a triple layer by using a $3 / 0$ barbed suture. The first layer involves Denonvilliers' fascia and the median raphe, the second the retrotrigonal fascia and the median raphe, and the third the bladder neck and the rhabdosphincter. The urethra-vesical anastomosis is made by using two 3/0 barbed hemi-running sutures, involving the full thickness of either the bladder or the urethra. The anterior reconstruction consists of two layers of $3 / 0$ barbed running sutures. The first layer involves the muscular fibres of the bladder and the peri-urethral tissue. The second involves the vesical apron and the portion of the endopelvic fascia that covers the dorsal vein complex while involving the pubo-prostatic ligaments.

sRARP was performed successfully in all the cases without the need of conversion. No major intraoperative complication occurred. All the patients underwent extended lymph node dissection and 3 patients (27.2\%) could benefit from a monolateral intermediate nerve-sparring according to Pasadena Classification ${ }^{[21]}$.

No differences in terms of technical surgical difficulties were found between patients undergoing primary focal or whole-prostate HIFU.

Median catheterization time was 5 days (IQR: 4-7). In 3 patients (27.2\%), cystography was performed in the 5th post-operative day, before catheter removal. Median hospital stay was 6 days (IQR: 6-8 days).

Focusing on safety, only Clavien 1 complications were recorded. One patient had postoperative fever treated with antibiotics infusion, and 1 patient had an asymptomatic lymphocele (about $4 \mathrm{~cm}$ in maximum diameter) detected at an ultrasound performed 3 months post-surgery.

On postoperative histopathology assessment, positive surgical margin rate was $27.2 \%$; in all of these cases, an extracapsular extension of the disease ( $\geq \mathrm{pT} 3$ ) was present and none of these patients underwent nervesparring. 


\section{DISCUSSION}

In case of local recurrence post-HIFU ablation, sRP is a treatment option for selected patients; however, there is a paucity of data concerning the perioperative, functional, and oncological outcomes of this procedure.

Literature about salvage RP after HIFU treatment is very limited and characterized by small series reporting heterogeneous populations including patients submitted to whole gland ablation and focal ablation ${ }^{[13-15,22,23]}$. Furthermore, the available studies concern a number of very different radical prostatectomy techniques (i.e., open, laparoscopic, and robotic).

A multi-institutional study by Marconi et al. ${ }^{[22]}$, presented the largest published series of sRARP in men experiencing recurrent disease after focal therapy (defined as the ablation of the index lesion using different energy sources) for PCa. The authors demonstrated that sRARP post focal therapy was safe and had excellent urinary continence outcomes (83.1\%), using a very strict continence definition (i.e., the use of no pads). These data are very similar to our series in which we observed $81.1 \%$ of urinary continence rate at 12 months.

However, it must be emphasized that our case report involved patients operated by a very experienced surgeon in a high-volume center. The achievement of such postoperative functional outcomes may not be reached in situations that do not reflect these requirements, therefore the recommendation of performing sRARP remains the prerogative of a few select centers.

Moreover, in Marconi et al. ${ }^{[22]}$ 's cohort, a high rate of biochemical recurrence was observed post-surgery, leading to a multimodal treatment approach in a considerable number of patients (41.1\%).

The authors observed that men experiencing an infield recurrence post-focal therapy had almost 5 times the chance of developing recurrence post sRARP, independent of the margin status, Gleason score, or pT stage.

In the present study, biochemical recurrence at 12 months was observed in only one patient, but it is important to underline the short oncological follow up.

In a retrospective matched pair analysis, the functional and oncological outcomes of sRARP, following recurrent disease after focal therapy (mostly HIFU), were compared with men undergoing RP as a primary treatment ${ }^{[23]}$. In this series, $53 \%$ of the patients submitted to sRARP were continent, using the "no pad" definition, and $31.8 \%$ presented with biochemical recurrence after surgery. When sRARP patients were matched with 44 patients undergoing primary RARP, no difference in urinary continence, perioperative outcomes, and complication rates were found. However, sRARP patients demonstrated a 4.8 -fold increased risk of biochemical recurrence when compared to primary RARP patients ${ }^{[23]}$.

Thompson et al. ${ }^{[14]}$ demonstrated that patients undergone sRARP post-partial ablation of the prostate using HIFU had acceptable perioperative outcomes overall, but with higher than expected rates of anastomotic leaks and bladder neck contractures. The continence rate was quite low $(66 \%)$ and the significant positive surgical margins (SM) rate was high (26\%), possibly, according to the authors, as a result of a greater proportion of focal re-treatments pre-sRARP in their series. No patient recovered erectile function at 3- or 12-month post-sRARP ${ }^{[14]}$. 
In our series positive, SM rate was quite similar (27.2\%), and in all of these cases extracapsular extension of the disease $(\geq \mathrm{pT} 3$ ) was present. Sexual function outcomes were poor also in our patients (potency rate $18 \%$ ). Although we have performed monolateral nerve-sparring surgery in 3 patients, we are convinced that potency preservation with monoliteral/bilateral nerve-sparring should not be a realistic aim of sRARP post HIFU for a combination of post HIFU fibrosis, cancer factors, and erectile dysfunction.

The major limitations of the present study are: the short oncological follow-up time and the retrospective design of the study. Important limitations, which also concerned the other few studies about this topic, include the absence of standardized criteria for focal therapy, salvage treatment, and the definition of focal therapy failure. Finally, it is very likely that patients selected for sRARP, instead of further focal treatment or surveillance, presented with more aggressive recurrences. As such, patients in the current study may not be representative of all men experiencing recurrent disease after focal therapy.

However, our findings are particularly noteworthy because we reported and classified the adverse events and the treatment impacts on patients' quality of life, rigorously filling a gap of the current literature ${ }^{[24]}$. Moreover, our data, despite the limited sample size, are encouraging and confirm that overall complications rate and urinary continence outcomes of sRARP for men experiencing disease recurrence post HIFU are acceptable and are not much worse than those in the primary setting.

\section{DECLARATIONS}

\section{Authors' contributions}

Conception and design: De Luca S, De Cillis S

Acquisition of data: De Cillis S, Piramide F, Alessio P, Volpi G, Granato S

Analysis and interpretation of data: De Luca S, De Cillis S, Checcucci E

Drafting of the manuscript: De Luca S, De Cillis S

Critical revision of the manuscript for important intellectual content: Porpiglia F, Fiori C, Manfredi M

Administrative, technical, or material support: Russo F

Supervision: Amparore D, Fiori C

\section{Availability of data and materials}

Not applicable.

\section{Financial support and sponsorship}

None.

\section{Conflicts of interest}

All authors declared that there are no conflicts of interest.

\section{Ethical approval and consent to participants}

This is to certify that for all patients who underwent HIFU, the focal treatment was approved by the local ethics committee (Candiolo cancer institute - FPO - IRCCS; registry number: 258/2018), and that all patients who underwent RARP after HIFU signed an informed consent.

\section{Consent for publication}

Not applicable 


\section{Copyright}

(C) The Author(s) 2022.

\section{REFERENCES}

1. Ferlay J, Soerjomataram I, Dikshit R, et al. Cancer incidence and mortality worldwide: sources, methods and major patterns in GLOBOCAN 2012. Int J Cancer 2015;136:E359-86. DOI PubMed

2. Cooperberg MR, Lubeck DP, Meng MV, Mehta SS, Carroll PR. The changing face of low-risk prostate cancer: trends in clinical presentation and primary management. J Clin Oncol 2004;22:2141-9. DOI PubMed PMC

3. Mottet N, van den Bergh RCN, Briers E, et al. EAU-EANM-ESTRO-ESUR-SIOG guidelines on prostate cancer-2020 update. Part 1: screening, diagnosis, and local treatment with curative intent. Eur Urol 2021;79:243-62. DOI PubMed

4. Schröder FH, Hugosson J, Roobol MJ, et al; ERSPC Investigators. Screening and prostate-cancer mortality in a randomized European study. N Engl J Med 2009;360:1320-8. DOI PubMed

5. De Luca S, Fiori C, Bollito E, et al. Risk of Gleason score 3 $+4=7$ prostate cancer upgrading at radical prostatectomy is significantly reduced by targeted versus standard biopsy. Minerva Urol Nefrol 2020;72:360-8. DOI PubMed

6. Bianchi L, Gandaglia G, Fossati N, et al. Oncologic outcomes in prostate cancer patients treated with robot-assisted radical prostatectomy: results from a single institution series with more than 10 years follow up. Minerva Urol Nefrol 2019;71:38-46. DOI PubMed

7. Ahdoot M, Lebastchi AH, Turkbey B, Wood B, Pinto PA. Contemporary treatments in prostate cancer focal therapy. Curr Opin Oncol 2019;31:200-6. DOI PubMed PMC

8. Valerio M, Cerantola Y, Eggener SE, et al. New and established technology in focal ablation of the prostate: a systematic review. Eur Urol 2017;71:17-34. DOI PubMed

9. Elkhoury FF, Simopoulos DN, Marks LS. MR-guided biopsy and focal therapy: new options for prostate cancer management. Curr Opin Urol 2018;28:93-101. DOI PubMed PMC

10. De Luca S, Fiori $\mathrm{C}$, Tucci $\mathrm{M}$, et al. Prostate cancer management at an Italian tertiary referral center: does multidisciplinary team meeting influence diagnostic and therapeutic decision-making process? Minerva Urol Nefrol 2019;71:576-82. DOI PubMed

11. Guillaumier S, Peters M, Arya M, et al. A multicentre study of 5-year outcomes following focal therapy in treating clinically significant nonmetastatic prostate cancer. Eur Urol 2018;74:422-9. DOI PubMed PMC

12. Stabile A, Orczyk C, Hosking-Jervis F, et al. Medium-term oncological outcomes in a large cohort of men treated with either focal or hemi-ablation using high-intensity focused ultrasonography for primary localized prostate cancer. BJU Int 2019;124:431-40. DOI PubMed

13. Clery R, Grande P, Seisen T, et al. Outcomes after salvage radical prostatectomy and first-line radiation therapy or HIFU for recurrent localized prostate cancer: results from a multicenter study. World J Urol 2019;37:1491-8. DOI PubMed

14. Thompson JE, Sridhar AN, Shaw G, et al. Peri-operative, functional and early oncologic outcomes of salvage robotic-assisted radical prostatectomy after high-intensity focused ultrasound partial ablation. BMC Urol 2020;20:81. DOI PubMed PMC

15. Linares Espinós E, Sánchez-Salas R, Sivaraman A, et al. Minimally invasive salvage prostatectomy after primary radiation or ablation treatment. Urology 2016;94:111-6. DOI PubMed

16. Panebianco V, Villeirs G, Weinreb JC, et al. Prostate magnetic resonance imaging for local recurrence reporting (PI-RR): international consensus-based guidelines on multiparametric magnetic resonance imaging for prostate cancer recurrence after radiation therapy and radical prostatectomy. Eur Urol Oncol 2021;4:868-76. DOI PubMed

17. Roach M 3rd, Hanks G, Thames H Jr, et al. Defining biochemical failure following radiotherapy with or without hormonal therapy in men with clinically localized prostate cancer: recommendations of the RTOG-ASTRO Phoenix Consensus Conference. Int J Radiat Oncol Biol Phys 2006;65:965-74. DOI PubMed

18. Porpiglia F, Bertolo R, Manfredi M, et al. Total anatomical reconstruction during robot-assisted radical prostatectomy: implications on early recovery of urinary continence. Eur Urol 2016;69:485-95. DOI PubMed

19. Manfredi M, Checcucci E, Fiori C, et al. Total anatomical reconstruction during robot-assisted radical prostatectomy: focus on urinary continence recovery and related complications after 1000 procedures. BJU Int 2019;124:477-86. DOI PubMed

20. Campobasso D, Fiori C, Amparore D, et al. Total anatomical reconstruction during robot-assisted radical prostatectomy in patients with previous prostate surgery. Minerva Urol Nefrol 2019;71:605-11. DOI PubMed

21. Montorsi F, Wilson TG, Rosen RC, et al; Pasadena Consensus Panel. Best practices in robot-assisted radical prostatectomy: recommendations of the Pasadena Consensus Panel. Eur Urol 2012;62:368-81. DOI PubMed

22. Marconi L, Stonier T, Tourinho-Barbosa R, et al. Robot-assisted radical prostatectomy after focal therapy: oncological, functional outcomes and predictors of recurrence. Eur Urol 2019;76:27-30. DOI PubMed

23. Nunes-Silva I, Barret E, Srougi V, et al. Effect of prior focal therapy on perioperative, oncologic and functional outcomes of salvage robotic assisted radical prostatectomy. J Urol 2017;198:1069-76. DOI PubMed

24. Checcucci E, Amparore D, De Luca S, Autorino R, Fiori C, Porpiglia F. Precision prostate cancer surgery: an overview of new technologies and techniques. Minerva Urol Nefrol 2019;71:487-501. DOI PubMed 